\title{
ARTICLE
}

Acute lymphoblastic leukemia

\section{Simultaneous B and T cell acute lymphoblastic leukemias in zebrafish driven by transgenic MYC: implications for oncogenesis and lymphopoiesis}

\author{
Chiara Borga ${ }^{1} \cdot$ Gilseung Park $^{1} \cdot$ Clay Foster $^{1} \cdot$ Jessica Burroughs-Garcia ${ }^{1} \cdot$ Matteo Marchesin ${ }^{1} \cdot$ Rikin Shah $^{1}$. \\ Ameera Hasan ${ }^{1}$ - Syed T. Ahmed ${ }^{1}$. Silvia Bresolin ${ }^{2}$. Lance Batchelor ${ }^{1} \cdot$ Teresa Scordino $^{3} \cdot$ Rodney R. Miles $^{4}$. \\ Geertruy te Kronnie $\mathbb{( I )}^{2} \cdot$ James L. Regens ${ }^{5} \cdot$ J. Kimble Frazer $\mathbb{( i D}^{1}$
}

Received: 13 March 2018 / Revised: 30 May 2018 / Accepted: 4 July 2018 / Published online: 15 August 2018

(c) The Author(s) 2018. This article is published with open access

\begin{abstract}
Precursor-B cell acute lymphoblastic leukemia (pre-B ALL) is the most common pediatric cancer, but there are no useful zebrafish pre-B ALL models. We describe the first highly- penetrant zebrafish pre-B ALL, driven by human MYC. Leukemias express B lymphoblast-specific genes and are distinct from T cell ALL (T-ALL)—which these fish also develop. Zebrafish pre-B ALL shares in vivo features and expression profiles with human pre-B ALL, and these profiles differ from zebrafish T-ALL or normal B and T cells. These animals also exhibit aberrant lymphocyte development. As the only robust zebrafish pre-B ALL model and only example where T-ALL also develops, this model can reveal differences between $M Y C$ driven pre-B vs. T-ALL and be exploited to discover novel pre-B ALL therapies.
\end{abstract}

\section{Introduction}

Acute lymphocytic leukemia (ALL), a common cancer and the most prevalent childhood malignancy, comprises $>25 \%$ of pediatric neoplasia in the U.S., with $\sim 85 \%$ being pre-B

These authors contributed equally: Gilseung Park, Clay Foster.

Electronic supplementary material The online version of this article (https://doi.org/10.1038/s41375-018-0226-6) contains supplementary material, which is available to authorized users.

$\triangle$ J. Kimble Frazer

Kimble-Frazer@ouhsc.edu

1 Section of Pediatric Hematology-Oncology, Department of Pediatrics, University of Oklahoma Health Sciences Center, Oklahoma City, OK 73104, USA

2 Department of Women's and Children's Health, University of Padua, Padua 35128, Italy

3 Department of Pathology, University of Oklahoma Health Sciences Center, Oklahoma City, OK 73104, USA

4 Department of Pathology, University of Utah and ARUP Institute for Clinical \& Experimental Pathology, Salt Lake City, UT 84108, USA

5 Center for Intelligence and National Security, University of Oklahoma, Norman, OK 73019, USA
ALL [1, 2]. Relapses are all-too-common, making ALL the highest cause of pediatric cancer-related death [3]. Thus, there is a dire need for animal models of pre-B ALL to identify new molecular targets and discover new therapies, but efforts are impeded by a lack of in vivo models amenable to genetic and drug screens.

Zebrafish (Danio rerio) provide one potential solution, since they can model human leukemias accurately [4], have practical advantages (genetic tractability, high-throughput screens, cost), and share hematopoietic, oncogenic, and tumor suppressive pathways with humans [5]. These features permitted the creation of several zebrafish T cell ALL (T-ALL) models that mimic the human disease [6-10], which subsequently led to key findings in T-ALL genetics, disease progression mechanisms, and signaling [11-16], as well as facilitating screens for new treatment agents $[17,18]$.

However, despite the even greater clinical impact of preB ALL, effective zebrafish models lag behind. A single report of zebrafish B-ALL using transgenic ETV6-RUNX1 [19] had low penetrance and long latency ( 3\% by 1 year), and no subsequent reports with this or any other B-ALL model exist.

Here we utilized a cell-specific fluorophore, lck:eGFP [20], that labels zebrafish $\mathrm{B}$ and $\mathrm{T}$ cells differentially to discover the first robust $D$. rerio B-ALL model. 
Surprisingly, B-ALLs occur in an already-established TALL model driven by transgenic human MYC [10], and they are so prevalent that many animals actually have coincident B-ALL and T-ALL. An intensive investigation of this new model using several approaches revealed a number of important findings. First, $h M Y C$-induced B-ALL are pre-B subtype, express immature B cell transcripts, and like human pre-B ALL, spread aggressively to lymphoid and non-lymphoid tissues. Second, pre-B ALL express low levels of $l c k$, and thus are dimly-fluorescent in these animals, unlike the brightly-fluorescent T-ALL of this model. Low $L C K$ expression is conserved in human pre-B ALL. Third, in addition to their differential lck:eGFP expression, we report a two-gene classifier that distinguishes pre-B from T-ALL in $h M Y C$ fish. Finally, expression profiles of zebrafish pre-B ALL, T-ALL, and normal $\mathrm{B}$ and $\mathrm{T}$ cells revealed abnormal lymphopoiesis that may underlie the molecular pathogenesis of $h M Y C$-driven ALL. In summary, we report a novel and robust pre-B ALL model, the first in zebrafish. Besides its value for genetic and drug screens, to our knowledge, $h M Y C$ fish represent the only animal model that develops both pre-B and T-ALL, providing a unique tool to explore molecular mechanisms of both human ALL types in the same genetic context, or even the same animal.

\section{Materials and methods}

Zebrafish care and microscopy, FACS and flow cytometry analysis, qRT-PCR, RNA-microarrays, H\&E, IHC, and WB analysis used standard techniques (see supplementary for details). Microarrays Data deposited at NCBI GEO repository GSE109437 (https://www.ncbi.nlm.nih.gov/geo/ query/acc.cgi?acc $=$ GSE109437).

\section{RNAscope-ultrasensitive in situ hybridization (RNA- ISH)}

RNAscope (Advanced Cell Diagnostics-ACD, Hayward, CA, USA) fluorescent-field ISH used to detect $h M Y C$, $c d 79 b$, and lat mRNA in fish sections. Procedure performed using the Multiplex-Fluorescent-Detection-Kitv2 (\#323110), according to manufacturer instructions (https://acdbio.com/). RNAscope probes used to specifically detect human MYC (\#311761-C2), D. rerio cd79b (\#511481) and lat (\#507681). Probe labels (PerkinElmer, Waltham, MA, USA) as follows: TSA-Plus-Cyanine-3 (\#NEL744001KT) for $h M Y C$ (yellow fluorescence), TSAPlus-Cyanine-5 (\#NEL745001KT) for $c d 79 b$ (red), and TSA-Fluorescein (\#NEL701A001KT) for lat (green). Slides imaged and analyzed using an Operetta HighContent Imaging System (PerkinElmer) and Harmony 4.1 software.

\section{Nanostring nCounter gene expression profiling}

GEPs of FACS-purified GFP ${ }^{\text {lo }}$ and GFP Gi $^{\text {hi }}$ cell populations were quantified using a 96-gene Custom CodeSet according to manufacturer instructions (Nanostring nCounter Technologies, Seattle, WA, USA). Genes quantified using an nCounter Digital Analyzer and analysed using nSolver v3.0 software. Background thresholds defined by counts from a no-RNA blank that were subtracted from each sample. Raw counts were normalized to spiked-in positive control probes and housekeeping genes ( $\beta$-actin, eeflall1, and gapdh), as suggested by the manufacturer. nSolver $t$ tests used to compare groups and identify differentiallyexpressed genes $(\mathrm{FDR} \leq 0.05)$.

\section{Results}

\section{Human MYC induces two zebrafish ALL types with distinct expression signatures}

Mammalian $M y c / M Y C$ transgenes driven by a $D$. rerio rag 2 promoter induce zebrafish T-ALL $[6,10]$. To detect and monitor ALL progression, we built double-transgenic fish by crossing $\operatorname{Tg}(r a g 2: h M Y C)$ to $\operatorname{Tg}(l c k: e G F P)$ fish, where a zebrafish lck promoter controls GFP expression [20].

Henceforth, we refer to this double-transgenic line as $h M Y C ; G F P$. The original report with $h M Y C$-transgenic fish utilized tamoxifen to augment MYC activity via its fusion to a modified estrogen receptor;[10] in our study tamoxifen was not used. To study T-ALL in our system, we performed RNA microarray on FACS-purified $\mathrm{GFP}^{+}$cells dissected from the bodies of $10 h M Y C$;GFP fish and 3 hlk fish [9], another zebrafish T-ALL model (see Fig. 1A for example animals).

Unsupervised analysis divided $h l k$ and $h M Y C ; G F P$ malignancies precisely, emphasizing fundamental differences in ALL from different $D$. rerio models (Fig. 1A). Unexpectedly, $h M Y C ; G F P$ ALL also clustered into two subgroups with distinct gene expression profiles (GEPs).

To further investigate these groups, we used hlk T-ALL as a reference and designated the 4 ALL closest to $h l k$ as hMYC-1, and the $6 \mathrm{ALL}$ at the far right as $h M Y C$-2 (blue and orange samples in Fig. 1A).

Separate comparisons of $h M Y C-1$ or $h M Y C-2$ vs. hlk ALL revealed that $\mathrm{B}$ cell-specific genes were up-regulated by both types of $h M Y C$; GFP ALL (pax5, btk, cd81, etc.; Fig. 1B), with $h M Y C-2$ ALL over-expressing additional B cell-specific genes (syk, blnk, ighm). Ingenuity Pathway Analysis $^{\mathrm{TM}}$ (IPA) of these differentially-expressed genes showed enrichment and activation of "PI3K-Signaling in B-

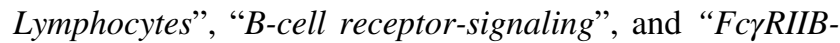
signaling in B-cells" pathways by hMYC-2 ALL, but not 

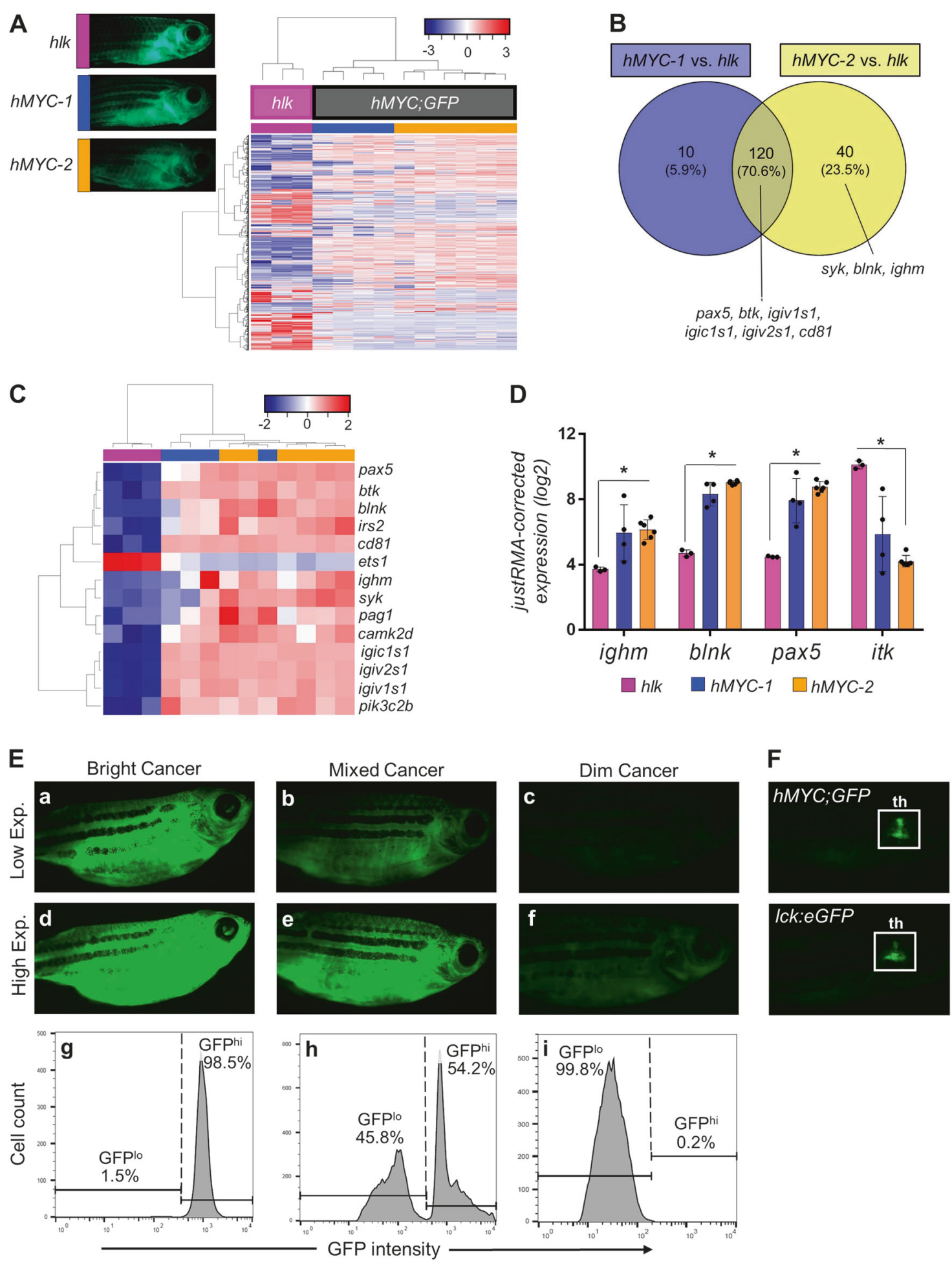

$h M Y C-1$, relative to hlk T-ALL (data not shown). To further investigate the unanticipated expression of B cell genes by hMYC ALL, we repeated unsupervised analysis using only 14 statistically-significant B cell-specific genes. Remarkably, this signature classified hlk vs. hMYC ALL perfectly and largely reformed both the $h M Y C-1$ and $h M Y C-2$ subclasses (Fig. 1C).
Expression of $\mathrm{B}$ cell genes by $h M Y C$ cancers was unexpected, because B-ALL has never been described by several laboratories-including ours-that study transgenic Myc/MYC zebrafish [6, 10, 11, 15, 18, 21]. Yet microarrays clearly demonstrated B cell genes (ighm, blnk, pax5) were expressed at high, medium, and low levels by $h M Y C-2$, $h M Y C-1$, and $h l k$ ALL, respectively, with T cell-specific itk 
Fig. 1 Two ALL types in $h M Y C$ zebrafish with differing fluorescence intensities. A Unsupervised analysis of $10 h M Y C$ (grey) and 3 $h l k$ (magenta) ALL, using highest-variance probes. $h M Y C$ ALL cluster into $h M Y C-1$ (blue) and $h M Y C-2$ (orange) groups. Representative fluorescent images of fish with ALL from each group shown at upper left. B Venn diagram of 170 over-expressed genes in $h M Y C$ ALL compared to $h l k$ T-ALL. Genes up-regulated by both $h M Y C-1$ and -2 $(n=120)$ reside in the intersection, including six B cell-specific genes listed below the Venn diagram. Three other B cell-specific genes overexpressed by only $h M Y C-2$ ALL are listed below the yellow circle. C Unsupervised analysis using B cell-specific genes. D Expression of ighm, blnk, pax5 and itk in hlk, hMYC-1 and $h M Y C-2$ ALL. Each gene is significantly differentially expressed in $h l k$ T-ALL vs. $h M Y C-2$ ALL (Mann-Whitney test, $* p$-value $<0.05$ ). Expression values are $\log _{2}$ scale, normalized against the entire microarray dataset using the justRMA algorithm. Results shown as mean values \pm standard deviation (S.D.). E Left: "bright" ALL, shown using low and high exposure settings (a, d). Cells are GFP hi by flow cytometry (g). Right: "dim" ALL, using low and high $(\mathbf{c}, \mathbf{f})$ exposures. Cells are GFP ${ }^{\text {lo }}(\mathbf{i})$. Center: Fish with mixed-ALL (b, e), with discrete GFP ${ }^{\text {lo }}$ and GFP ${ }^{\text {hi }}$ populations (h). F Images of control $h M Y C ; G F P$ (upper) and lck: $e G F P$ (lower) fish with only thymic (th) fluorescence

showing the opposite pattern (Fig. 1D). We hypothesized hMYC- 1 and $h M Y C-2$ cancers might contain not only TALL cells, but also different fractions of B lymphocytes, accounting for these findings.

Specifically, we predicted that $h l k$ cancers were "pure" T-ALL, whereas $h M Y C-1$ contained some B cells but mostly T-ALL cells, and that $h M Y C-2$ samples contained the highest percentage of B and/or B-ALL cells relative to T-ALL cells. Alternatively, leukemias can express aberrant markers [22], and $h M Y C$ might de-differentiate ALL, obscuring cell identities. In either case, B cell genes were highest in $h M Y C-2$ and detectable in $h M Y C-1$ also, so we next sought to definitively identify the cellular composition of $h M Y C$ cancers.

\section{B-ALL and T-ALL each occur in hMYC;GFP animals, with different GFP intensities}

To definitively identify $h M Y C ; G F P$ ALL as they first developed, we used serial fluorescent microscopy to monitor unaffected animals (i.e., fish lacking visible cancers). In young adults (3-6 months), we observed two phenotypes: brightly-fluorescent cancers originating in thymus and dimly-fluorescent cancers with variable thymic involvement (Fig. 1E, S1). To distinguish these, we used "low-exposure" settings that detected only bright cancers [Fig. 1E(a) vs. (c), S1A vs. S1B], and "high-exposure" settings that revealed dim ALL which were otherwise not visible [Fig. 1E(c) vs. (f), S1B vs. S1D]. Dim ALL differed from non-cancerous $h M Y C$ and lck:eGFP control animals that showed only normal thymic fluorescence (Fig. 1F).

Microscopy findings were confirmed by dissecting $\mathrm{GFP}^{+}$ tissue from the bodies of these animals for flow cytometric analysis of $\mathrm{GFP}^{+}$cells from the lymphocyte/precursor gate
[23]. Bright and dim ALL showed distinct, $>10$-fold GFP intensity differences [Fig. 1E(g-i)]. Thus, we could discern ALL with only bright ( $>90 \% \mathrm{GFP}^{\text {hi }}$ cells), only dim $(>90 \%$ $\mathrm{GFP}^{\text {lo }}$ cells), or mixed cell populations $(>10 \%$ for either minority $\mathrm{GFP}^{\text {hi }}$ or $\mathrm{GFP}^{\mathrm{lo}}$ population). We analyzed ALL from $27 h M Y C$ fish with fluorescent cancers at 6 months of age and found $7 \mathrm{dim}$ ALL with near-exclusively $\mathrm{GFP}^{\mathrm{lo}}$ cells (Fig. 2A) and 14 GFP $^{\text {hi }}$-only ALL (Fig. 2C). Intriguingly, we also found 6 mixed-ALL that contained distinct populations of both GFP ${ }^{\text {hi }}$ and GFP ${ }^{\text {lo }}$ cells (Fig. 2B). Remarkably, these 27 animals developed 33 total ALL, 13 GFP $^{\text {lo }}$ and $20 \mathrm{GFP}^{\text {hi }}$. Of note, mixed ALL exhibited varying amounts of $\mathrm{GFP}^{\mathrm{lo}}(23-76 \%)$ and $\mathrm{GFP}^{\text {hi }}(24-77 \%)$ populations; we attribute this to different onset dates and growth rates for each cancer. Thus, we predict these ratios change over time as each ALL independently progresses.

We next tested FACS-purified $\mathrm{GFP}^{+}$dim, bright, or mixed-ALL cells for B cell- ( $p a x 5, c d 79 b$, ighz, etc.), T cell( $c d 4, c d 8, i l 7 r$, etc.), and lymphoblast- (rag2, igicls 1 , etc.) specific transcripts, as well as the GFP and $h M Y C$ transgenes by quantitative reverse-transcriptase PCR (qRT$\mathrm{PCR}$ ), analyzing all $\mathrm{GFP}^{+}$cells as one population without separating $\mathrm{GFP}^{\mathrm{lo}}$ and $\mathrm{GFP}^{\mathrm{hi}}$ peaks. Dim/GFP ${ }^{\text {lo }}$ ALL expressed B, but not T, cell-specific genes [Fig. 3A(a-d), $\mathrm{S} 2 \mathrm{~A}(\mathrm{a}-\mathrm{d})]$. Low $l c k$ and GFP levels in dim ALL matched their weak in vivo fluorescence. Conversely, bright/GFP ${ }^{\text {hi }}$ cancers expressed only $\mathrm{T}$ cell genes. Mixed-ALL expressed both $\mathrm{B}$ and $\mathrm{T}$ cell genes at intermediate levels. Overall, expression correlated exactly with $\mathrm{dim} / \mathrm{GFP}^{\mathrm{lo}}$ vs. bright/GFP ${ }^{\text {hi }}$ phenotypes, and only mixed-ALL, which contained $\mathrm{GFP}^{\mathrm{lo}}$ and $\mathrm{GFP}^{\text {hi }}$ cells, co-expressed genes of both cell types. Based on these findings, we conclude $\mathrm{dim} /$ GFP $^{\text {lo }}$ ALL are B-lineage ALL. Mixed-ALL always exhibited distinct $\mathrm{GFP}^{\mathrm{lo}}$ and $\mathrm{GFP}^{\mathrm{hi}}$ cell populations (Fig. 2B) and expressed B-lineage and T-lineage genes [Fig. 3A(a-d), S2A(a-d)], so we deduce mixed-ALL are not biphenotypic, but simultaneous B-ALL and T-ALL in one animal.

B-lineage vs. T-lineage ALL could be unambiguously distinguished by igic1s1 [Fig. 3A(e), S2A(e)], a predicted homologue of the IGLL1 surrogate Ig light chain gene expressed by only immature B cells [24]. Only dim and mixed-ALL expressed igicls1, but every ALL showed similar levels of the $\mathrm{V}(\mathrm{D}) \mathrm{J}$ recombination enzyme rag2 [Fig. 3A(f), S2A(f)]. Based on igicls1 and rag2 results, which only immature B cells co-express, we believe $h M Y C$ B-ALL are an immature B cell cancer, most likely pre-B ALL. A zebrafish rag2 promoter regulates $h M Y C$, so it is logical that $\mathrm{rag}^{+}$B-lymphoblasts (i.e., pre-B cells) are affected, just like T-ALL in this model $[6,10]$. Moreover, similar $h M Y C$ levels in pre-B and T-ALL [Fig. 3A(f), S2A (f)] indicate this transgene has similar oncogenic potency in both lymphocyte lineages. Surprisingly, ragl was much 

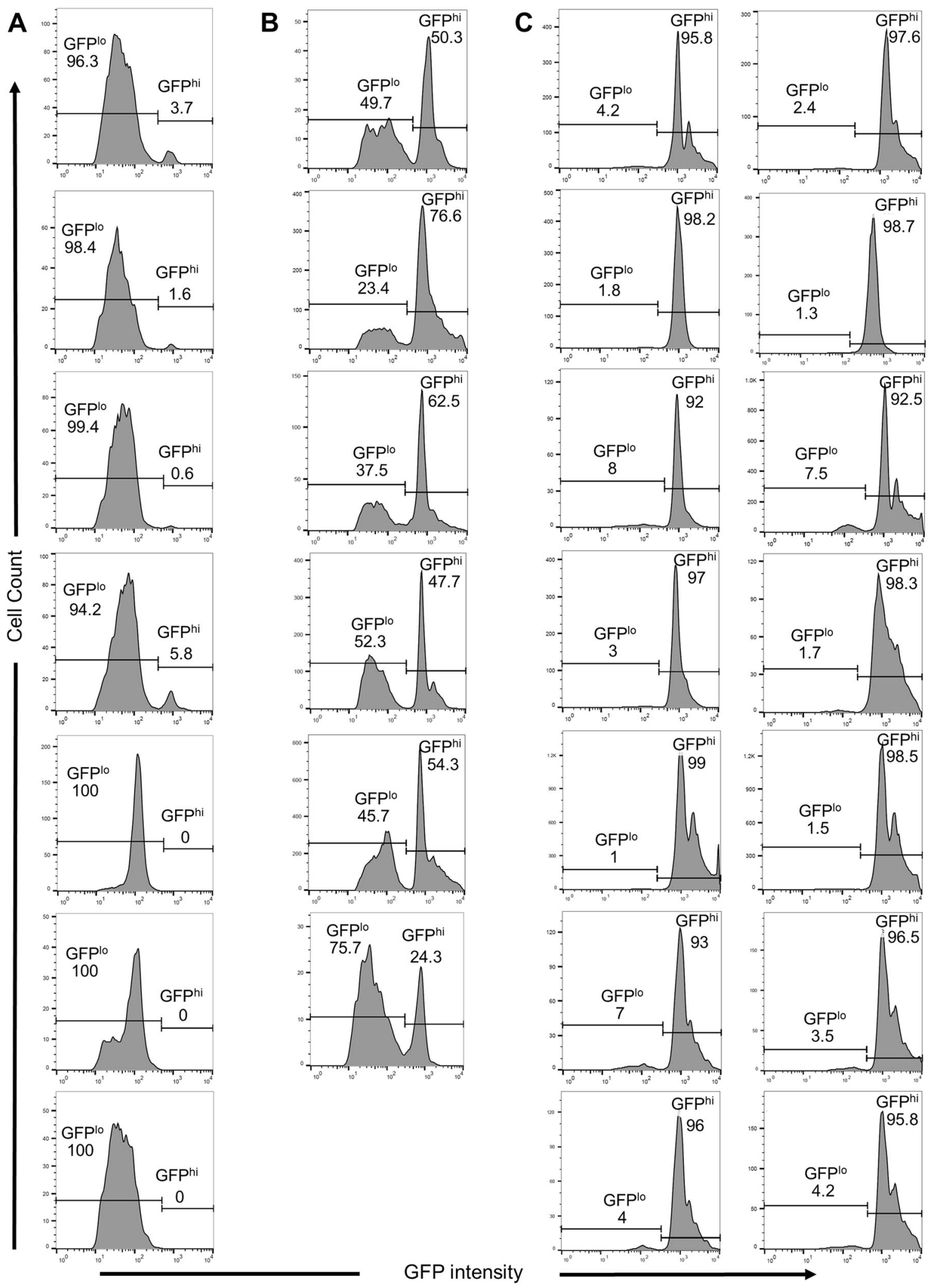

Fig. 2 Distinct GFP intensities of $h M Y C$ dim and bright ALL. Flow cytometric plots of 33 ALL from 27 6-month $h M Y C$ fish: A 7 dim, GFP ${ }^{\text {lo }}$ ALL, B 6 mixed, GFP ${ }^{\text {lo }} \& \mathrm{GFP}^{\text {hi }}$ ALL, and C 14 bright, GFP ${ }^{\text {hi }}$ ALL 
A $\square$ dim $\square$ mixed $\square$ bright
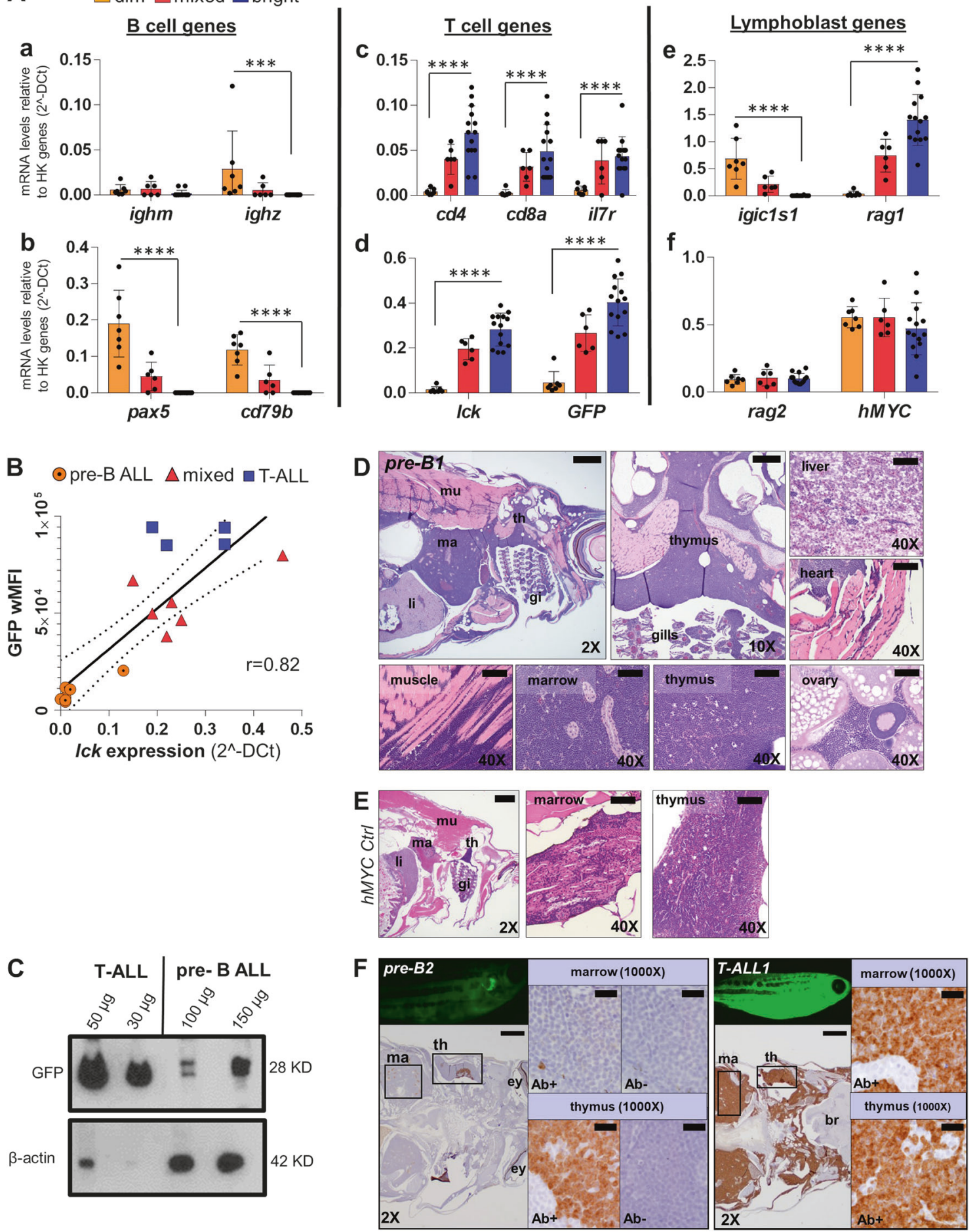

Fig. 3 GFP fluorescence intensity of $h M Y C$ ALL correlates with B vs. $\mathrm{T}$ cell lineage. A qRT-PCR of ALL with differing GFP fluorescence (dim, $n=7$; mixed, $n=6$; bright, $n=14$ ) of B cell-specific (ighm, $i g h z, \operatorname{pax} 5, c d 79 b ; \mathbf{a}-\mathbf{b})$, T cell-specific $(c d 4, c d 8 a, i l 7 r, l c k ; \mathrm{c}-\mathrm{d})$, lymphoblast- specific (igic1s1, rag1, rag2; e-f) genes and transgenes $(G F P, \mathrm{~d} ; h M Y C, \mathrm{f})$. Results are normalized to housekeeping (HK) genes $(\beta$-actin and eeflall1) and shown as mean values \pm S.D. Significant differences are indicated (Mann-Whitney test, $p$-values: $* * *<0.001, * * * *<0.0001)$. B Spearman correlation ( $p$-value 0.0002 , $r=0.82, r^{2}=0.74$ ) between $l c k$ vs. WMFI for: 7 B-ALL (circles),
6 mixed (triangles), and 4 T-ALL (squares). The solid line represents linear regression; dashed lines denote $95 \%$ confidence intervals. C Anti-GFP (\#sc-9996) and anti- $\beta$-actin (\#ab8227) WB of FACSpurified T- and pre-B ALL. D H\&E of pre-B ALL infiltration in different sites and $\mathbf{E} h M Y C$ control. F Anti-GFP (\#GTX20290) IHC of $h M Y C$ pre-B ALL (pre-B2, left) and T-ALL (T-ALL1, right). 1000× images show staining with or without anti-GFP $(\mathrm{Ab}+; \mathrm{Ab}-)$. th= thymus, $\mathrm{ma}=$ marrow, $\mathrm{li}=$ liver, $\mathrm{mu}=$ muscle, $\mathrm{gi}=$ gills, ey= eye, $\mathrm{br}=$ brain. $2 \times$ scale bar $=500 \mu \mathrm{m} ; 10 \times$ bar $=100 \mu \mathrm{m} ; 40 \times$ bar $=50 \mu \mathrm{m}$; $1000 \times$ bar $=20 \mu \mathrm{m}$ 
A

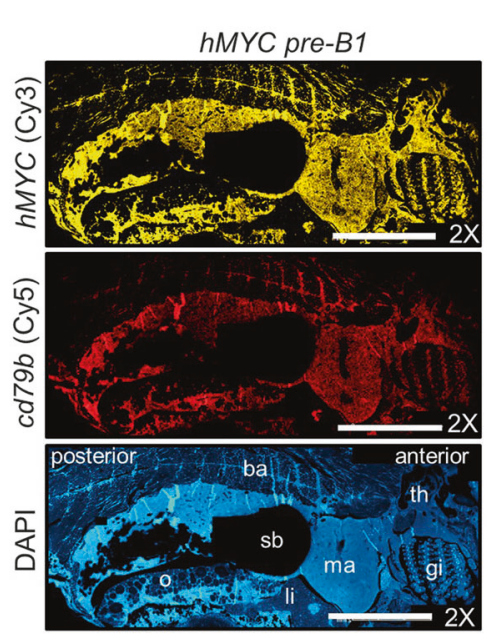

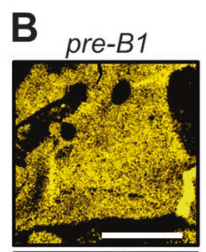
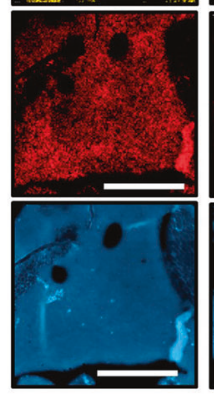

D
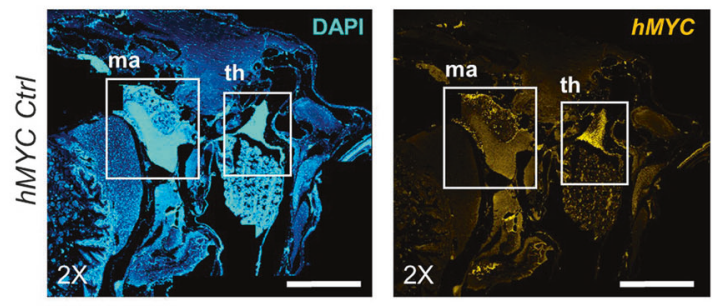
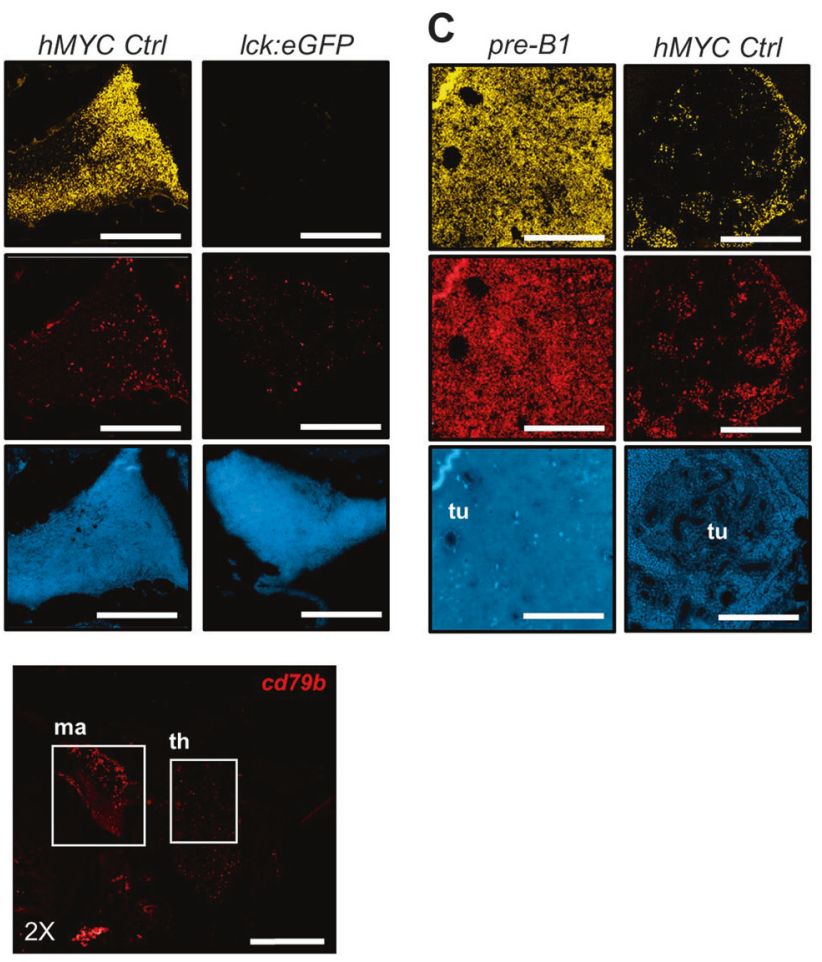

E

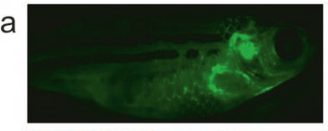

b
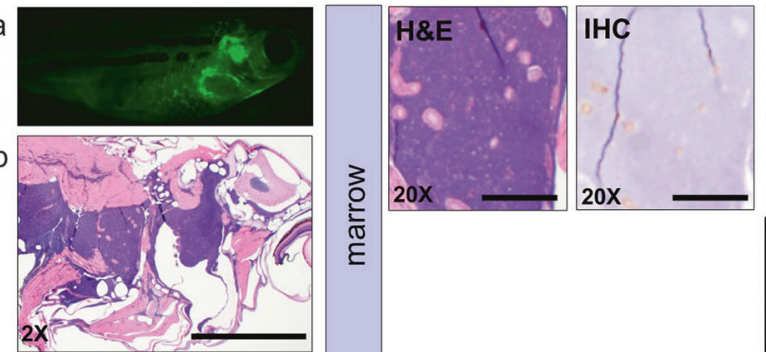

C
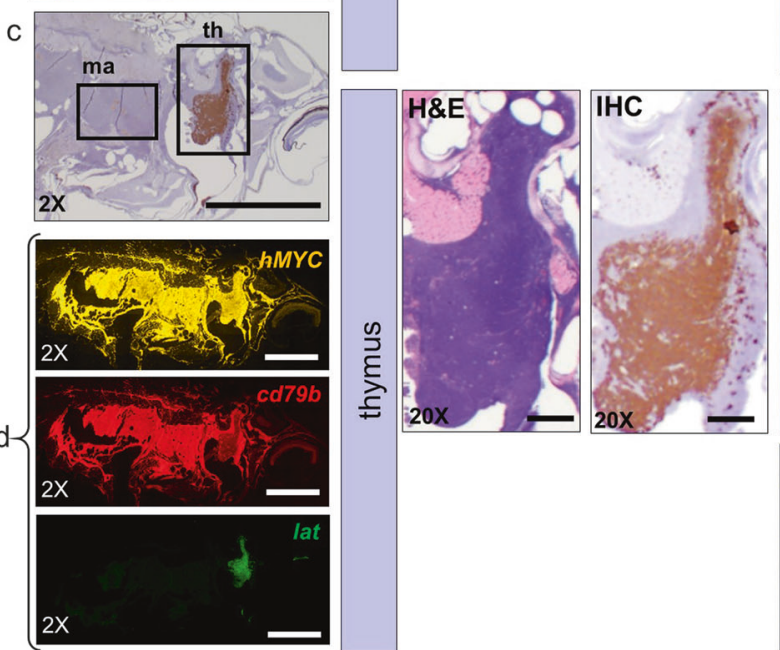

lower in pre-B ALL [Fig. 3A(e), S2A(e)], making igicls 1 and ragl a two-gene panel that can distinguish $h M Y C$ ALL types independent of $l c k$ or GFP levels. Mammalian Blymphoblasts and T-lymphoblasts co-express RAG1 and RAG2, so dichotomous ragl levels were unexpected.
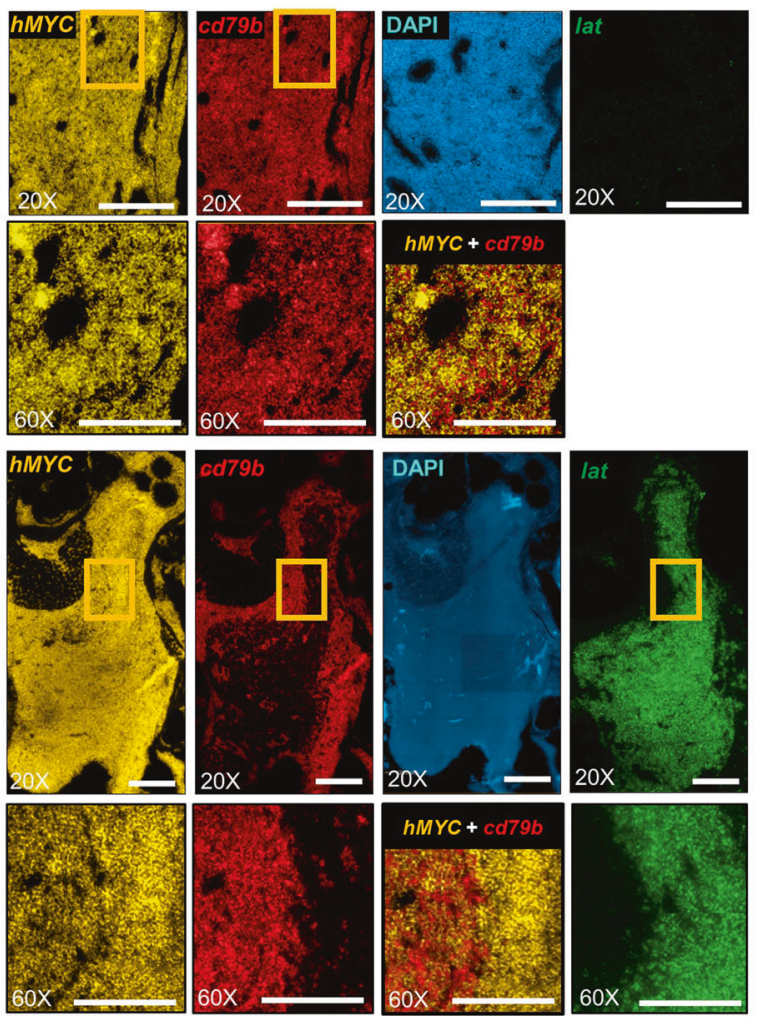

However, unlike mammals, rag2-mutant zebrafish lack $\mathrm{T}$ cells, yet retain functional B cells [25]. How D. rerio B cells develop in this context is unknown, because if ragl is absent in B cells, only mutant Rag2 would be present to mediate immunoglobulin (Ig) recombination. Potential 
Fig. 4 Pre-B ALL co-express $h M Y C$ and B cell-specific $c d 79 b$. RNA ISH for $h M Y C$ (yellow) and $c d 79 b$ (red) in: A sagittally-sectioned hMYC pre-B ALL (pre-B1; scale bar $=2 \mathrm{~mm}$ ), B Thymi of pre-B1 (left), $h M Y C$ control (center), and lck:GFP (WT) control (right; scale bar $=200 \mu \mathrm{m}), \mathbf{C}$ Kidney-marrow of pre-B1 (left) and $h M Y C$ control (right). Kidney tubules (tu) are displaced by pre-B1 ALL cells in marrow DAPI image (scale bar $=200 \mu \mathrm{m}$ ), D $h M Y C$ control (scale bar $=1 \mathrm{~mm}$ ). E Second $h M Y C$ fish with pre-B ALL and localized thymic T-ALL. Left: (a) High-exposure microscopy, (b) H\&E, (c) anti-GFP IHC, (d) RNA ISH for $h M Y C$ (yellow), $c d 79 b$ (red) and lat (green). Middle: high-power of kidney-marrow (top) and thymus (bottom) by H\&E, anti-GFP IHC. Right: $h M Y C, c d 79 b$, and lat, RNA ISH. Boxed regions in 20X marrow and thymus panels are enlarged in the $60 \times$ images directly beneath them. Merged $h M Y C+c d 79 b$ images are also shown. $2 \times$ scale bar $=2 \mathrm{~mm} ; 20 \times$ bar $=200 \mu \mathrm{m} ; 60 \times$ bar $=100 \mu \mathrm{m}$. Abbreviations as in Fig. 3, and $\mathrm{o}=$ ovary, $\mathrm{ba}=\mathrm{back}, \mathrm{sb}=\mathrm{swim}$ bladder

explanations for this paradox include compensatory ragl expression by B cells, expression of ragl at a different B cell stage, hypomorphic function of the truncated mutant Rag2 protein, or the formal possibility that zebrafish utilize a different $\mathrm{V}(\mathrm{D}) \mathrm{J}$ recombination mechanism than mammals.

As predicted by different in vivo fluorescence [Fig. 1E (a-f), S1] and GFP ${ }^{\mathrm{lo}}$ vs. GFP ${ }^{\text {hi }}$ cytometric results [Fig. 1E (g-i), 2], lck and GFP also differed markedly between pre-B and T-ALL [Fig. 3A(d), S2A(d)]. A zebrafish $l c k$ promoter regulates GFP [20], and pre-B ALL expressed little $l c k$ or $G F P$, while T-ALL expressed both abundantly. In agreement, lck mRNA correlated with weighted median fluorescence intensity (wMFI; Fig. 3B), with each ALL type clustering separately, proving $l c k$ levels-and thus, cellular fluorescence-distinguish pre-B vs. T-ALL in this model. We also confirmed GFP mRNA and protein levels agree by Western blot (WB), with much higher amounts of total protein needed to detect GFP in pre-B ALL compared to TALL (Fig. 3C).

lck is generally considered to be $\mathrm{T}$ cell-specific [20], but zebrafish NK and myeloid cells also express lck [26, 27]. Pertinent to our study, we analyzed public data from different maturation stages of human lymphocytes [28] and found pre-B cells expressed higher $L C K$ than naïve and mature $\mathrm{B}$ cells, although below that of $\mathrm{T}$ cells (Fig. S2B). Furthermore, Microarray Innovations in Leukemia (MILE) data [29] from human patients showed that although mean $L C K$ levels are higher in T-ALL than pre-B ALL (Fig. S2C), 37\% of T-ALL (64/174 patients) expressed equivalent $L C K$ to $83 \%$ of pre-B ALL cases (409/493; red box in Fig. S2C), demonstrating $L C K$ is not specific to T-ALL.

This is further exemplified by comparing the highest $L C K$-expressing pre-B ALL quartile to the lowest T-ALL quartile in this dataset, for which no statistical difference exists ( $p$-value $=0.75$; Fig. S2D). Thus, human and zebrafish pre-B ALL both express $L C K / l c k$.

\section{Zebrafish pre-B ALL resembles human pre-B ALL morphologically}

To examine pre-B ALL histology, we analyzed $h M Y C$ fish with $\operatorname{dim}$ ALL. Hematoxylin and eosin (H\&E) stains showed lymphoblast infiltration of the kidney-marrow, thymus, liver, and elsewhere (Fig. 3D, S3A). Marrow hypertrophy was often profound, with marrow expansion and invasion through muscle into subcutaneous tissue and skin (Fig. S3A, pre-B3, -B4). Yet despite these massive disease burdens, fish remained only dimly fluorescent. As in humans, pre-B and T-ALL were indistinguishable by $\mathrm{H} \& \mathrm{E}$ (Fig. S3A-B), and both were markedly abnormal compared to control animals (Fig. 3E, S3C).

Immunohistochemical analysis (IHC), however, could discriminate pre-B from T-ALL, with very faint anti-GFP staining in $\mathrm{GFP}^{\mathrm{lo}}$ ALL vs. strong signals in $\mathrm{GFP}^{\text {hi }}$ ALL (Fig. $3 \mathrm{~F}$, pre-B2 vs. T-ALL1, S4A-B), and only remnant thymic tissue showing strong signals in pre-B ALL fish (Fig. 3F, pre-B2 and S4A, pre-B5, -B6). Consistent with this, regions stained weakly by anti-GFP $(\mathrm{Ab}+)$ corresponded to dimly-fluorescent anatomic regions (Fig. 3F, S4A; 1000X panels).

Because $\mathrm{Ab}$ recognizing zebrafish lymphocyte proteins are not available, we used RNA in situ hybridization (ISH; RNAscope $^{\mathrm{TM}}$ ) to independently test cell identities using probes for $h M Y C$ and B cell-specific $c d 79 b$ (Fig. 4A-C). hMYC labeled pre-B ALL strongly (Fig. 4A; H\&E of this animal shown in Fig. 3D), including cells in the thymus and kidney-marrow (Fig. 4B, C, pre-B1). These same areas were also $c d 79 b$-positive, confirming B-lineage. Thymi of $h M Y C$ control fish were avidly $h M Y C^{+}$, but had few $c d 79 b^{+}$cells (Fig. 4B, D, hMYC Ctrl), indicating thymic B cells are sparse unless pre-B ALL is present. Similarly, $h M Y C$ control marrow had fewer dually $h M Y C^{+} / c d 79 b^{+}$cells (Fig. 4C, D), with normal kidney-marrow architecture, including renal tubules. $h M Y C$ was absent in the thymus of control lck:eGFP fish (Fig. 4B, lck:eGFP) proving probe specificity, and showed rare $c d 79 b^{+} \mathrm{B}$ cells, demonstrating few thymic B cells in WT fish.

We examined a different animal with disseminated preBALL and localized T-ALL, based on microscopy and IHC findings (see microscopy, IHC, and H\&E in Fig. S4A, pre-B6), adding a T-cell-specific probe, lat, to distinguish pre-B vs. TALL. RNA ISH demonstrated cells that were $h M Y C^{+}$/ $c d 79 b^{+} /$lat had completely replaced the marrow and thymic cortex (Fig. 4E), with $\mathrm{GFP}^{\text {hi }} h M Y C^{+} / c d 79 b^{-} / l a t^{+}$cells remaining only in an enlarged thymic medulla (i.e., localized T-ALL).

Similar results were seen in a second animal with nearcomplete thymic ablation by pre-B ALL (Fig. S4C; Fig. 3F, pre-B2 shows microscopy and IHC of this specimen). 
Additional RNA ISH for $c x c r 4 a$, another gene up-regulated in $h M Y C$ pre-B vs. T-ALL (Fig. S5A), showed identical staining to $c d 79 b$ and opposite staining to lat in two different specimens (Fig. S5B-C), demonstrating $h M Y C$ ${ }^{+} / c d 79 b^{+} / c x c r 4 a^{+} / l a t^{-}$cells correspond to B-lineage $\mathrm{GFP}^{\text {lo }}$ cancers. In summary, in vivo fluorescence, cytometric GFP intensity, qRT-PCR, WB, and RNA ISH all prove dim cancers in $h M Y C$; GFP fish are pre-B ALL with organ distributions similar to human pre-B ALL.

\section{Zebrafish pre-B ALL remain GFP ${ }^{l o} /{ }^{1 / 0} k^{\text {in }}$ every tissue and can be allo-transplanted}

Pre-B ALL disseminated aggressively (Fig. 3D-F, 4, S3A, S4A, C, S5B-C). To test whether pre-B ALL cells retained a $\mathrm{GFP}^{\mathrm{lo}} / l c k^{\mathrm{lo}}$ phenotype in every niche, we examined thymus, marrow, spleen, blood, and muscle and viscera by flow cytometry (Fig. 5). Fish with pre-B ALL $(n=5, \mathrm{~B} 1-5)$ showed GFP ${ }^{\text {lo }}$ cells in each tissue, with GFP $^{\text {hi }}$ cells (i.e., normal $\mathrm{T}$ cells) primarily present only in thymus. As in mammals, zebrafish thymus is the normal site of $\mathrm{T}$ cell development, explaining its high content of $\mathrm{GFP}^{\text {hi }}$ cells. Despite this, some fish (e.g., B1, B2) showed considerable $\mathrm{GFP}^{\mathrm{lo}}$ populations in thymus, which we consider to be preB ALL with thymic invasion. In contrast, fish M1 exhibited a range of $\mathrm{GFP}^{\mathrm{lo}}(37-97 \%)$ and $\mathrm{GFP}^{\text {hi }}(3-64 \%)$ cells in each tissue, with both populations abundant in non-lymphoid muscle and viscera, where $\mathrm{B}$ and $\mathrm{T}$ cells are typically scarce. Therefore, we conclude M1 had mixed-ALL, with widespread pre-B ALL, as well as a distinct T-ALL that had spread to muscle and viscera, but not marrow or peripheral blood. In contrast, hMYC T-ALL controls $(n=3$, T1-3) exhibited near-exclusively $\mathrm{GFP}^{\text {hi }}$ populations in every tissue.

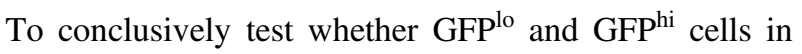
every anatomic site always represent B-lineage vs. Tlineage cell identities, we analyzed igiclsl and ragl in FACS-purified $\mathrm{GFP}^{\mathrm{lo}}$ and $\mathrm{GFP}^{\mathrm{hi}}$ cells from five of these fish (B1, B2, M1, T1, T2). As previously [Fig. 3A(e), S2A(e)], dim cells from every tissue expressed only igicls 1 , and only bright cells were $\operatorname{ragl} 1^{+}$, including the $\mathrm{GFP}^{\mathrm{lo}}$ and $\mathrm{GFP}^{\mathrm{hi}}$ ALLs of M1. Thus, GFP reliably reflects pre-B vs. T-ALL in any niche, establishing $h M Y C ; G F P$ zebrafish as a new and novel model to study both ALL types in one genetic context, or even one animal.

To test whether $h M Y C$ pre-B ALL were truly malignant, we transplanted FACS-purified GFP $^{\text {lo }}$ cells into sublethally-irradiated immunocompromised wild-type (WT) host fish. Multiple pre-B ALL engrafted $(n=9 / 11)$, remained $\mathrm{GFP}^{\mathrm{lo}}$, and continued to express only B-lineage markers (Fig. S6A). As reported for Myc-induced T-ALL [30, 31], we also serially transplanted $h M Y C$ pre-B ALL, which likewise retained GFP $^{\mathrm{lo}}$ B cell identities (Fig. S6A).
Interestingly, pre-B ALL exhibited lower leukemiainitiating cell (LIC) frequencies compared to those described for T-ALL [13, 30], although this may relate to the inherent difficulty of detecting engraftment by dimlyfluorescent cancers (Fig. S6B). Overall, these results indicate that GFP $^{\mathrm{lo}}$ pre-B ALL are malignant, and that their cellular identities are phenotypically stable.

\section{MYC-induced zebrafish pre-B and T-ALL have distinct expression signatures}

To test whether D. rerio and human pre-B ALL share similar gene expression, we next defined GEPs in a new cohort of animals, quantifying 96 transcripts (93 experimental genes plus 3 housekeeping controls) that distinguish $\mathrm{B} / \mathrm{T} / \mathrm{NK}$ cells, lymphoblasts, and precursor populations (genelist in Table S1) [31, 32]. We FACS-purified 8 pre-B ALL, 4 T-ALL, and 2 ALL from a mixed-ALL fish (Fig. S7A-C; GFP $^{\text {lo }}$ or GFP ${ }^{\text {hi }}$ populations in orange or blue, respectively), as well as control lymphocytes. GFP ${ }^{\text {hi }}$ or GFP $^{\mathrm{lo}}$ ALL purifications contain mostly malignant cells, but control lymphocytes are less homogeneous; we predicted GFP $^{\text {hi }}$ lymphocytes would be "T-cell enriched" and GFPlo cells "B-enriched." To test the accuracy and reproducibility of GFP-based sorting, we re-analyzed FACS-purified thymic and marrow lymphocytes from WT and $h M Y C$ fish by flow cytometry, confirming GFP ${ }^{\text {lo }}$ and $\mathrm{GFP}^{\text {hi }}$ cells are highly enriched for these populations (Table S2).

For $\mathrm{T}$ cell controls, we isolated $\mathrm{GFP}^{\text {hi }}$-enriched thymocytes from three 10-fish cohorts of control $h M Y C$ and lck:GFP fish (hMYC thymus-GFP ${ }^{\text {hi }}$, WT thymus$\mathrm{GFP}^{\text {hi }}$; Fig. S7D). As another T cell control, we pooled lymphoid-gated [23] $\mathrm{GFP}^{\text {hi }}$-enriched marrow cells from these same 30 WT fish (WT marrow-GFPi; Fig. S7E, blue). hMYC marrow lacked GFP ${ }^{\text {hi }}$ cells (Fig. S7F), so these were not analyzed. For B cells, we purified GFP ${ }^{\text {lo }}$ marrow cells from the same $3 h M Y C$ control cohorts ( $h M Y C$ marrow-GFP ${ }^{\mathrm{lo}}$; Fig. S7F) and from marrow of the 30 WT fish used for thymocyte preparations (WT marrow$\mathrm{GFP}^{\mathrm{lo}}$; Fig. S7E, orange). In addition, we also analyzed GFP $^{-}$cells from WT and $h M Y C$ marrow control samples (WT marrow-GFP'; $h M Y C$ marrow-GFP', Fig. S7E-F), to investigate which cell lineage(s) were enriched in this subfraction.

Using barcoded gene-specific probes (Nanostring nCounter ${ }^{\mathrm{TM}}$ ), we quantified mRNA levels of 93 transcripts in all 29 samples. Unsupervised analysis clustered all GFP ${ }^{\mathrm{lo}}$ and $\mathrm{GFP}^{\text {hi }}$ triplicate controls tightly ( $h M Y C$ thymus-GFP ${ }^{\text {hi }}$, WT thymus-GFP ${ }^{\text {hi }}$, $h M Y C$ marrow-GFP', $h M Y C$ marrow$\mathrm{GFP}^{\mathrm{lo}}$ ), proving high reproducibility of biologic replicates (Fig. 6A). In addition, each of the 29 samples segregated unambiguously as B-enriched or T-enriched, with every $\mathrm{GFP}^{-} / \mathrm{GFP}^{\mathrm{lo}}$ sample $(n=17)$ expressing B-lineage 

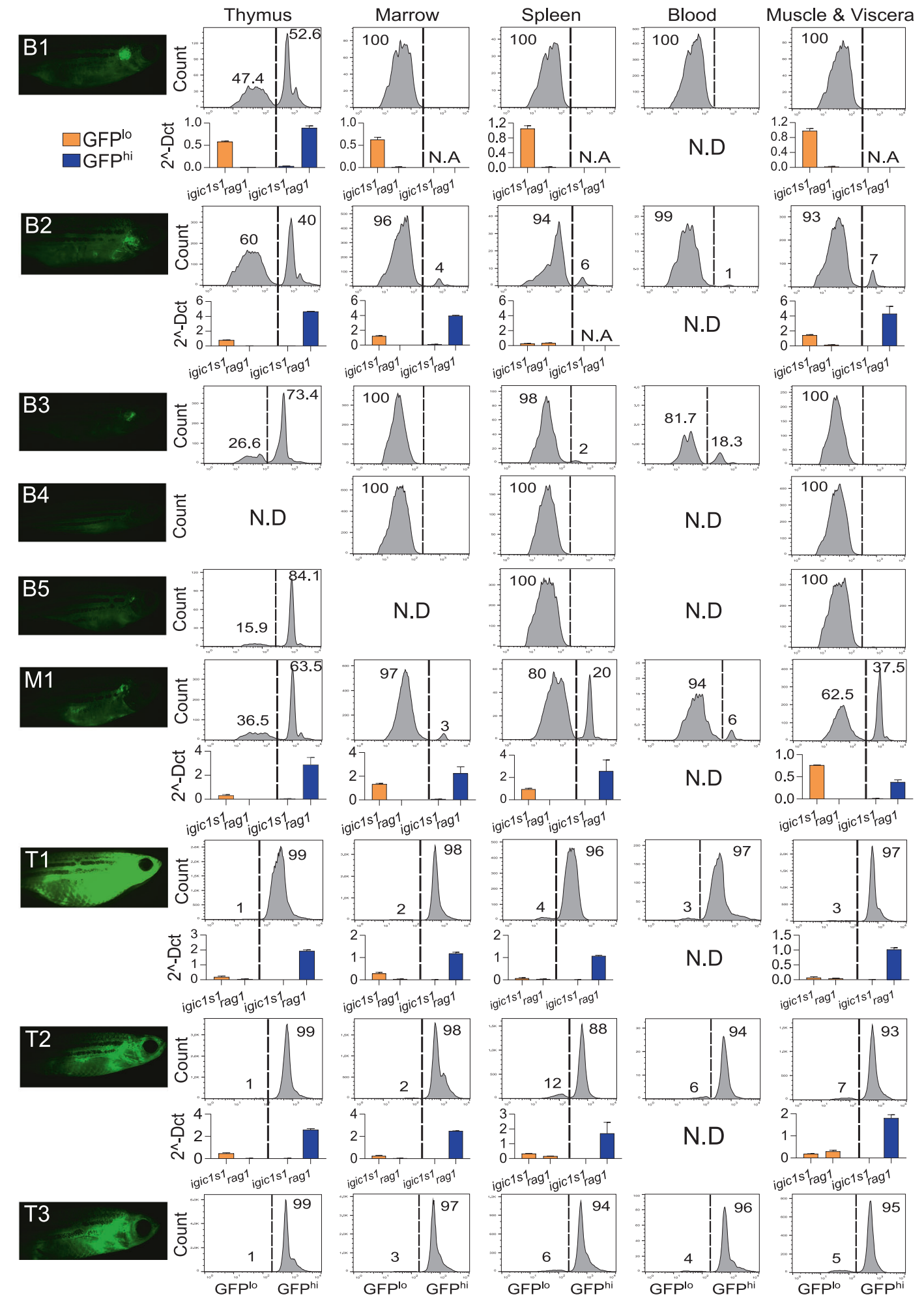

$\mathrm{igic}^{1} \mathrm{~s}^{1} \mathrm{rag}^{1} \mathrm{igic}^{1} \mathrm{Cs}^{1} \mathrm{rag}^{1}$

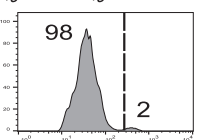

N.D
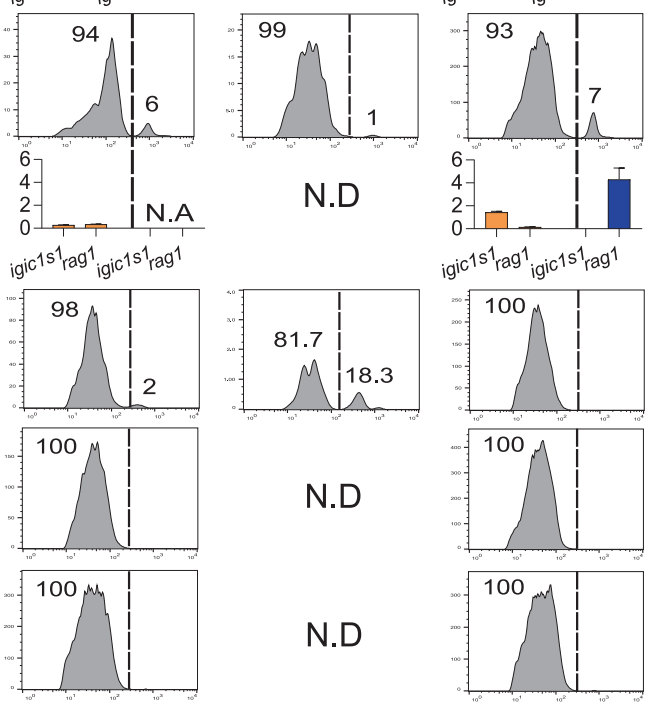

N.D
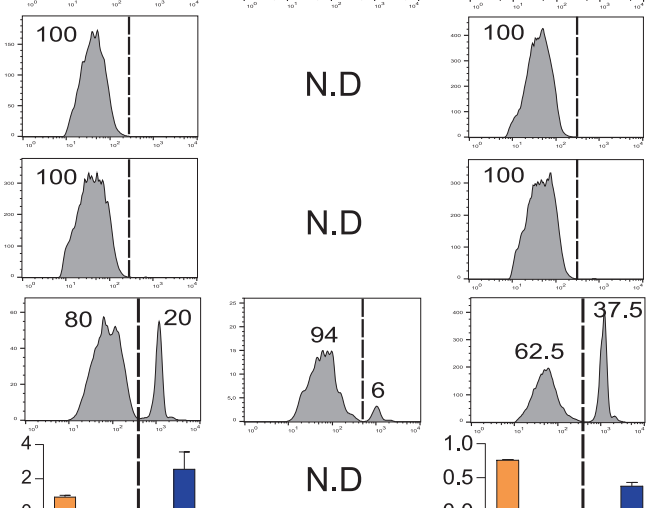

\section{N.D}
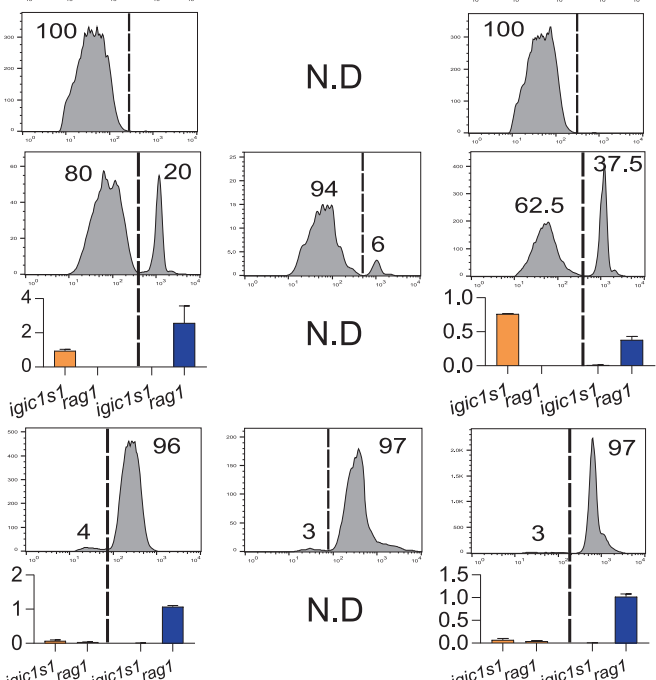

N.D
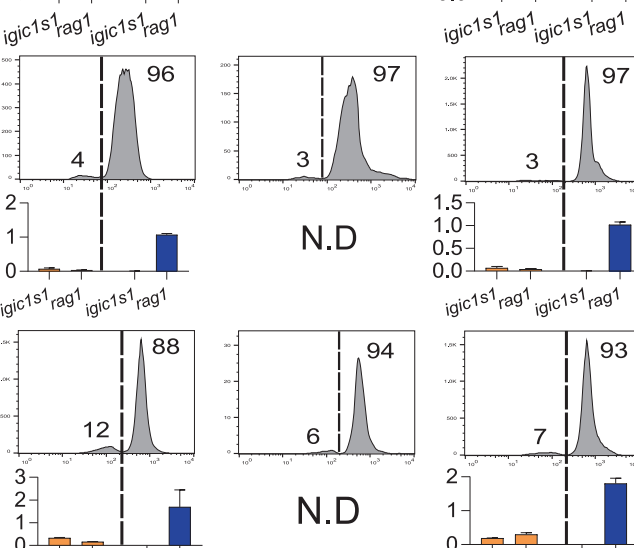

N.D
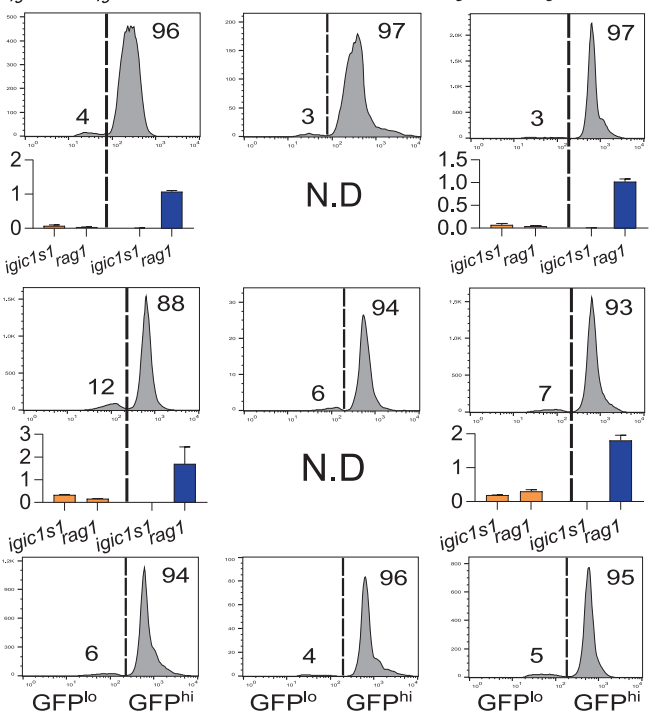

Fig. 5 GFP intensity and igic1s1/ragl distinguish pre-B vs. T-ALL in each anatomic site. Left column shows high-exposure fluorescent microscopy of 4 month-fish with pre-B ALL (B1-5), mixed ALL (M1), or T-ALL (T1-3). Panels at right show flow cytometric analysis of GFP $^{\text {lo }}$ and GFP ${ }^{\text {hi }}$ cells of thymus, marrow, spleen, peripheral blood, and muscle \& abdominal viscera. Each panel shows \% of $\mathrm{GFP}^{\mathrm{lo}}$ vs. $\mathrm{GFP}^{\text {hi }}$ cells in the entire $\mathrm{GFP}^{+}$gate; $10^{5}$ events from the lymphoid/

precursor gate were analyzed for each plot. N.D.= not determined. Histograms depict expression of igiclsl and ragl by qRT-PCR in five $h M Y C$ fish with pre-B ALL (B1, B2), mixed ALL (M1) or T-ALL (T1, $\mathrm{T} 2$ ). Results are normalized to housekeeping genes ( $\beta$-actin and eefla1l1) and shown as mean + Standard Error (S.E.). N.A. $=$ not available due to insufficient cells for RNA extraction 


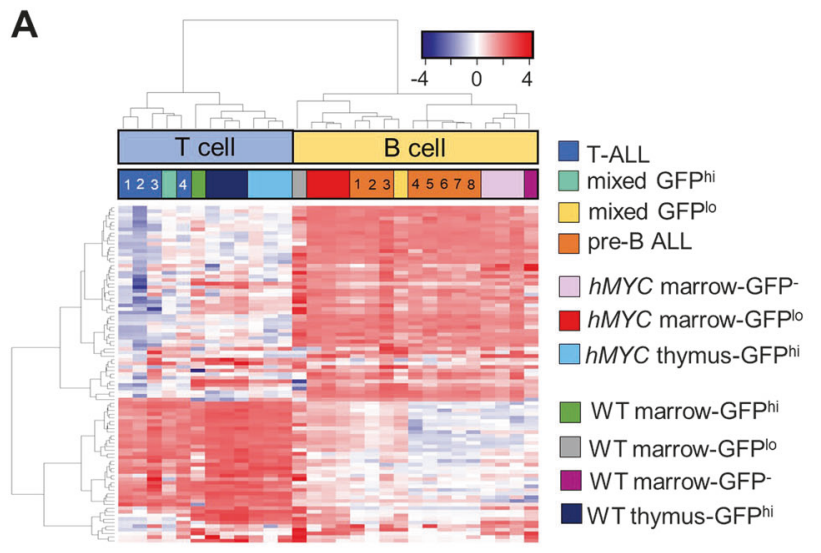

B

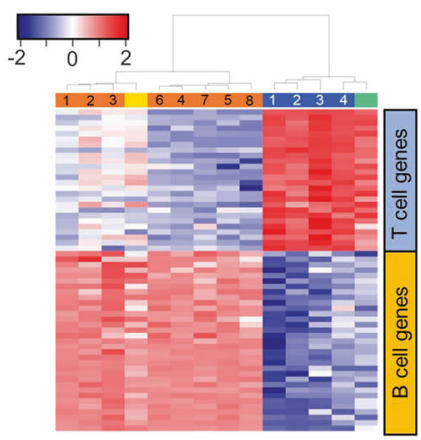

C
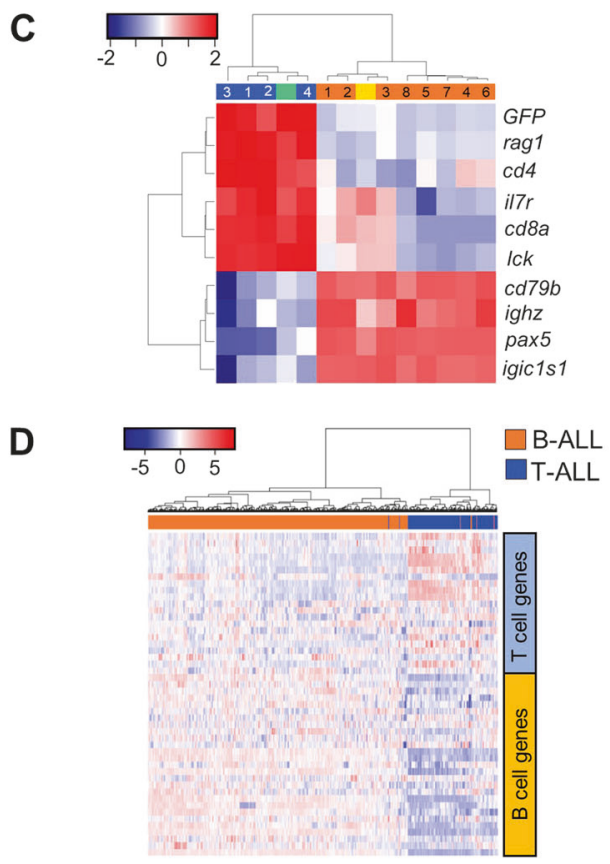

transcripts, and all GFP ${ }^{\text {hi }}$ samples $(n=12)$ enriched for T-lineage gene expression. This illustrates that GFP ${ }^{\text {hi }}$ control lymphocytes are highly enriched for T cells, while GFP ${ }^{-}$ and $\mathrm{GFP}^{\mathrm{lo}}$ populations are both enriched for B cells. This was true both for control fish or fish with ALL, irrespective of whether cells were from thymus or marrow, proving the value of $l c k: G F P$ in this system. Notably, GFP ${ }^{-}$and $\mathrm{GFP}^{\text {lo }}$ cells from WT and $h M Y C$ animals all exhibited similar B-

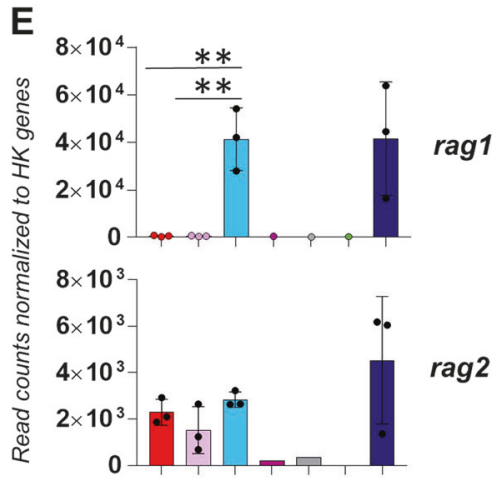

$\square$ hMYC marrow-GFP- $\square$ WT marrow-GFPhi hMYC marrow-GFPlo $\square$ WT marrow-GFPlo $\square$ hMYC thymus-GFPhi $\square$ WT marrow-GFPWT thymus-GFPhi
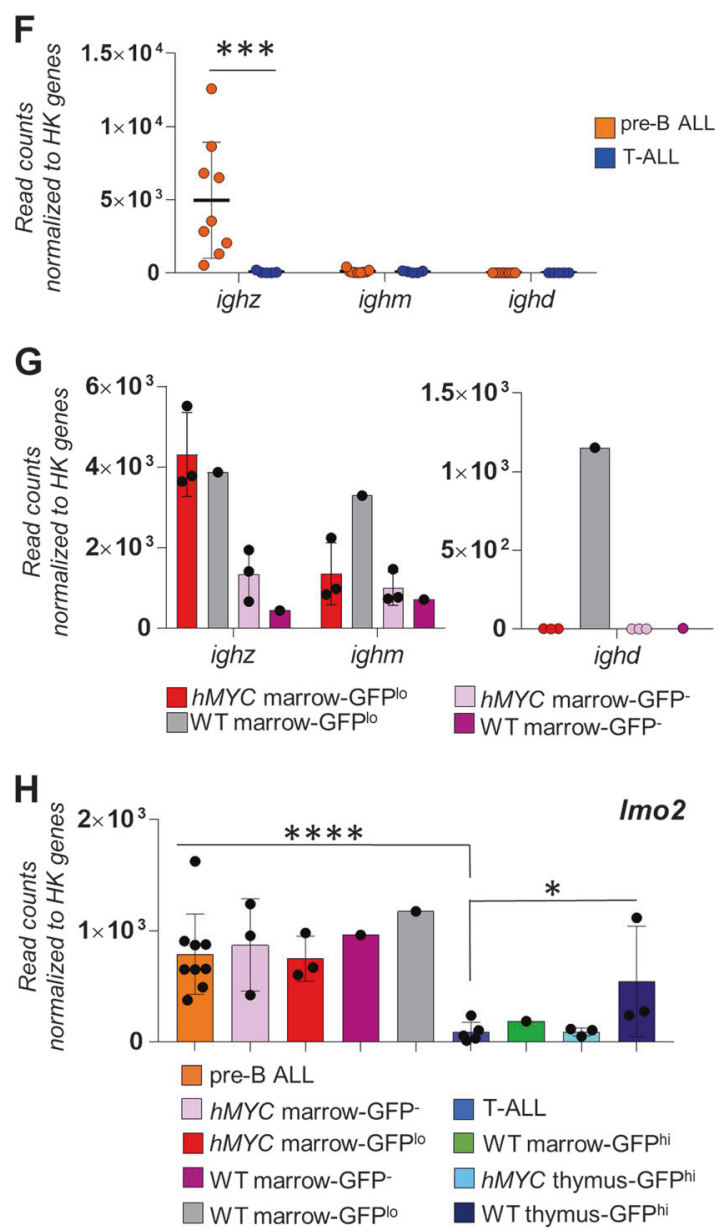

lineage profiles, with $h M Y C \mathrm{GFP}^{\mathrm{lo}} \mathrm{B}$ cell GEPs most similar to pre-B ALL (Fig. 6A).

Pre-B and T-ALL GEPs were distinct (Fig. 6B; Table S3 lists differentially-expressed genes), with the GFP ${ }^{\text {hi }}$ ALL (green) and GFP $^{\text {lo }}$ ALL (yellow) of the mixed-ALL animal grouping as T- or pre-B ALL, respectively. In total, $\sim 60$ homologous genes able to distinguish human [32] and zebrafish [31] B vs. T cells likewise categorized $h M Y C$ pre-B vs. T- 
Fig. $6 h M Y C$ drives pre-B and T-ALL with distinct GEP and alters Blineage expression. A Unsupervised analysis of all malignant and normal lymphocyte populations $(n=29)$ based on GEPs of 93 experimental genes ( $\beta$-actin, eefla1l1, gapdh housekeeping genes used for normalization not shown). Each sample groups as T- (blue box at top; $n=12$ ) or B-lineage (yellow box; $n=17$ ). Gene order listed in column D of Table S1. B Supervised analysis using significant $(\mathrm{FDR}<0.05)$ genes $(\mathrm{n}=59$; order in Table S3) distinguishing pre-B vs. T-ALL. Pre-B (orange; $\mathrm{n}=8$ ), T- (blue; $n=4$ ), and two ALL from a mixed-ALL $\left(\mathrm{GFP}^{\mathrm{lo}}=\right.$ yellow, $\mathrm{GFP}^{\mathrm{hi}}=$ green $)$ cluster as pre-B or $\mathrm{T}$ ALL. C Analysis with genes from prior qRT-PCR testing (Fig. 3A, S2A). D Unsupervised clustering of human ALL from patients on the MILE1 study, using homologues of zebrafish genes that distinguish $h M Y C$ pre-B vs. T-ALL (human genes in same order as Table S3). E rag1 (top) and rag2 (bottom) expression in $h M Y C$ and WT control B and T cell populations. F ighz, ighm and ighd levels in pre-B (orange) and T-ALL (blue). G ighz, ighm and ighd expression in GFP ${ }^{\text {lo }}$ (red) or $\mathrm{GFP}^{-}$(pink) non-malignant $h M Y C$ B cells, and $\mathrm{GFP}^{\text {lo }}$ (grey) or $\mathrm{GFP}^{-}$ (fuchsia) WT B cells. H lmo2 levels in all samples $(n=29)$ showing highest expression in B-lineage groups and lowest expression in TALL. E-H Mean values are shown \pm S.D., after normalization to $\beta$ actin, eefla1l1, and gapdh housekeeping genes. Significant differences noted (Mann-Whitney test, $p$-values: $*<0.05, * *<0.01, * * *<0.001$, $* * * *<0.0001)$

ALL. Key classifier-genes (Fig. 6C) matched prior qRT-PCR results (Fig. 3A, S2A), with both ALL types showing comparable levels of rag2, $h M Y C$, and shmt2, a known direct MYC target (Fig. S8A) [21], reinforcing that $h M Y C$ levels and activity are similar in this dual pre-B/T-ALL model. To further examine conservation of gene expression between both types of $D$. rerio and human ALL, we also tested whether human homologues of the differentially-expressed $h M Y C$ pre-B- vs. TALL genes could reliably classify ALL from the MILE1 study (Fig. 6D). This signature exhibited remarkable classification power, separating nearly all of these human ALL correctly. Homologues of several other hematopoietic stem/progenitor- or immature lymphocyte-specific genes showed no significant differences between $h M Y C$ ALL types (Fig. S8B).

Like ALL, control $h M Y C$ and WT thymocytes expressed more ragl than B cell controls (Fig. 6E, top). From this result, we deduce higher ragl in zebrafish T-ALL vs. pre-B ALL is unrelated to malignancy, but a normal feature of $D$. rerio lymphoblasts, although different from mammals.

Notably, $h M Y C$ B cell controls showed higher rag2 than WT B cells, implying $h M Y C$ may expand the pre-B cell population (Fig. 6E, bottom). We also analyzed Ig heavy chain expression.

Surprisingly, pre-B ALL expressed only ighz (Fig. 6F), an isotype unique to teleost fish that is functionally analogous to mammalian IgA [33]. GFP ${ }^{\text {lo }} h M Y C$ B cells expressed ighm (Fig. 6G, left), so ighm $^{+}$pre-B ALL should be detectable in hMYC fish-but these were not found. Therefore, MYC may be oncogenic in only the ighz-lineage. Another B-lineage abnormality we noted is that $h M Y C \mathrm{GFP}^{\mathrm{lo}}$ cells lacked ighd (Fig. 6G, right), so hMYC may suppress ighd transcription or repress this lineage, just as it seems to block the $\mathrm{T}$ cell lineage in $h M Y C$ marrow (Fig. S7F). Overall, $h M Y C$ dramatically perturbs zebrafish B cell development, inducing ALL in ighz, $\mathrm{B}$ cells, and skewing both the ighm and ighd lineages.

Finally, we found substantially higher lmo2 in normal and malignant $\mathrm{B}$ vs. T cells (Fig. 6H), mirroring LMO2 findings in human B vs. T cells [34], which we independently confirmed in public human $\mathrm{B}$ and $\mathrm{T}$ cell data (Fig. S8C). This contradicts the assertion that MYC-driven zebrafish T-ALL emulates the human TAL1/LMO subtype [6]. Instead, $h M Y C$ T-ALL actually had the lowest lmo2 levels of the nine populations that were tested, including all $\mathrm{T}$ cell controls (Fig. 6H).

\section{Discussion}

Pre-B ALL is the most common pediatric cancer and kills more children than any other type [3], but no good zebrafish models exist for this important disease. Drug screens [17, 18], genetic studies [11, 12, 15] and stem cell discoveries $[13,14]$ were all made possible by zebrafish $\mathrm{T}$ ALL models, and these and other approaches would likely be similarly fruitful with $D$. rerio pre-B ALL. Here, we describe the first robust zebrafish pre-B ALL model. Unexpectedly, young 3-6 month $h M Y C$ fish-used for years in several of the aforementioned T-ALL studies-also develop highly-penetrant pre-B ALL.

Remarkably, this went unrecognized for over a decade, raising the possibility that some prior T-ALL studies may have included mixed ALL (see Fig.1). Notably, in lines using the rag2:GFP marker instead of $l c k: G F P[6,10]$, pre$\mathrm{B}$ and T-ALL would be predicted to have identical fluorescence. Conversely, in other work using $l c k: G F P$ as a marker [31], unless investigators intentionally purified $\mathrm{GFP}^{\mathrm{lo}}$ cells, B cells may have been omitted, since B cell GFP is $<10 \%$ of that in $\mathrm{T}$ cells. In fact, even fish with massive pre-B ALL disease burdens exhibited barelydetectable fluorescence (Fig. S3A). Crucially, our data demonstrate that MYC can drive both T-lineage and Blineage leukemogenesis. This is not surprising, as human B and $\mathrm{T}$ cell leukemias all express $M Y C$ at high levels ( $p$ value $=0.34$; Fig. S2E). Recent studies likewise demonstrate key roles for MYC in B-lineage ALL molecular pathogenesis [35, 36].

In terms of detecting pre-B ALL, dual-transgenic $h M Y C ; G F P$ fish proved particularly valuable to our study, because their lck:GFP expression not only allowed pre-B ALL to be detected, but their differing GFP levels also distinguished pre-B and T-ALL in vivo. This dichotomy in $l c k$ expression extends to normal B and $\mathrm{T}$ cells as well, is corroborated by flow cytometry, and corresponds precisely to B-lineage and T-lineage GEPs (Fig. 1E, 2-6, S1, S4). Consequently, even in fish with concomitant pre-B and T-ALL-which we believe are 
unique to this model-these cell lineages and ALL types, can be reliably separated for independent study.

Apart from the utility of $l c k: G F P$ in this system, $h M Y C$ pre-B ALL are powerful because they emulate this human disease in several ways: histology and organ involvement (Figs. 3, 4, S3-5), lck levels (Fig. S2B-D), presence of LIC (Fig. S6) and, most importantly, gene expression signature (Fig. 6). In fact, genes that differentiate zebrafish pre-B vs. T-ALL (Fig. 6B) classify human ALL also (Fig. 6D). The GEPs we obtained revealed many transcripts that distinguish pre-B vs. T-ALL in this model, but we also report a two-gene panel to categorize $h M Y C$ ALL that requires only igiclsl and ragl, and we note this panel can be applied to any $h M Y C$ genetic background.

Interestingly, although $R A G 1$ is expressed by mammalian B-lymphoblasts and T-lymphoblasts, we find that only D. rerio T-lymphoblasts express ragl highly, with levels $>70$ - and $>155$-fold greater in normal T or T-ALL cells than in B or pre-B ALL cells, respectively (Fig. 3A(e), S2A(e), $5,6 \mathrm{C}, \mathrm{E})$.

A recent report of zebrafish B cell development despite low ragl supports our observation [37]. Apart from ragl, pre-B ALL expressed other classic B-lymphoblast genes like rag2, surrogate light chain components, $p a x 5, c d 79 a / b$, and others (Figs. 3, 4, 6C, S4C, S5B-C, S8A-C). This was true for every dim ALL, including GFP ${ }^{\text {lo }}$ mixed-ALL, so we conclude mixed-ALL are co-existing pre-B and T-ALL, and not biphenotypic ALL. Certainly, because hMYC can induce both ALL types, it remains possible that mixedlineage biphenotypic ALL may arise in this system, but to date, our analyses of $>40$ dim ALL have failed to detect any that express $\mathrm{T}$ cell genes, suggesting that GFP ${ }^{\mathrm{lo}}$ ALL are always B-lineage, and GFP ${ }^{\mathrm{hi}}$ always represent T-ALL. In future work, studying both ALL types in one backgroundor one animal-presents new opportunities, like efforts to find cooperating genetic lesions unique to one type of ALL, lymphocyte lineage-specific drugs, or myriad other applications.

Our results also demonstrate multiple features of abnormal B and T lymphopoiesis in $h M Y C$ fish. Of interest, pre-B ALL GEPs closely matched the gene expression pattern of a recently-described $\mathrm{igh}^{+} \mathrm{B}$ cell population dubbed "fraction 2" [37], suggesting $h M Y C$ is oncogenically active in this cell population. Supporting this, every pre-B ALL we identified was $i g h z^{+}$(Fig. 6F). Liu et al. postulated that the zebrafish ighm-lineage lacks a classic pre-B stage. If this is correct, it is logical that pre-B ALL only occurs in $i g h z^{+}$ cells, the lineage that has pre-B cells. We note that $\mathrm{ighm}^{+}$ cells do express lck (Fig. 6G), so $i g h m^{+}$pre-B ALL should be $\mathrm{GFP}^{+}$and detectable in this system. Yet $i g h m^{+}$pre-B ALL were never detected, so we conclude they do not occur. In addition to our finding that ALL develops in only $i g h z^{+}$cells, we also found that $h M Y C$ alters other B- lineages, with ighm and ighd both reduced in $h M Y C$ marrow (Fig. 6G). Whether this is due to ighm and ighd transcriptional-or lineage-repression remains to be determined. Notably, T cells are also diminished in $h M Y C$ marrow (Fig. S7F, Table S2), so $h M Y C$ alters both the B and $\mathrm{T}$ lymphocyte lineages, beyond inducing both pre-B and TALL.

Clearly, $h M Y C$ is leukemogenic to lymphocytes and perturbs zebrafish lymphocyte development. In future work, hMYC pre-B ALL can be used for classic zebrafish approaches like chemical and genetic screens, or in mechanistic studies probing $h M Y C$ biology in either ALL type. MYC is arguably the most clinically-relevant oncogene, important in many cancers besides ALL [38], but MYC's contrasting actions in distinct neoplasias remain largely unexplored. We show $h M Y C$ fish provide a novel system to address this topic via studies of both human ALL types, using a single model.

Acknowledgements We thank Megan-Malone Perez at OUHSC for zebrafish care, Sheryl Tripp at ARUP Laboratories for tissue sectioning and IHC, and Drs. Stephan Ladisch, David Jones, and Linda Thompson for critically reading the manuscript, and Dr. Nikolaus S. Trede for his support at the project's outset. An Institutional Development Award (IDeA) from the National Institute of General Medical Sciences (P20 GM103639) supports the OUHSC Stephenson Cancer Center's Histology and Immunohistochemistry Core, which performed services for this project.

Author contributions $\mathrm{CB}$ and JKF conceived and designed the research study. CB, CF, JLR, and JKF analyzed the data. CB, GP, MM, STA, JBG, RS, and AH performed experiments. TS and RRM performed histologic analyses and imaging. GtK, JLR, and JKF contributed essential reagents, tools and/or funding. SB and LB assisted with data analyses. CB and JKF wrote the manuscript. All authors revised and approved the final manuscript.

Funding JKF received support from Hyundai Hope On Wheels, the Oklahoma Center for the Advancement of Science and Technology (HRP-067), and an INBRE pilot project award from the National Institute of General Medical Sciences (P20 GM103447), and holds the E.L. \& Thelma Gaylord Endowed Chair of the Children's Hospital Foundation.

\section{Compliance with ethical standards}

Conflict of interest The authors declare that they have no conflict of interest.

Open Access This article is licensed under a Creative Commons Attribution 4.0 International License, which permits use, sharing, adaptation, distribution and reproduction in any medium or format, as long as you give appropriate credit to the original author(s) and the source, provide a link to the Creative Commons license, and indicate if changes were made. The images or other third party material in this article are included in the article's Creative Commons license, unless indicated otherwise in a credit line to the material. If material is not included in the article's Creative Commons license and your intended use is not permitted by statutory regulation or exceeds the permitted use, you will need to obtain permission directly from the copyright 
holder. To view a copy of this license, visit http://creativecommons. org/licenses/by/4.0/.

\section{References}

1. Jabbour E, O'Brien S, Konopleva M, Kantarjian H. New insights into the pathophysiology and therapy of adult acute lymphoblastic leukemia. Cancer. 2015;121:2517-28.

2. Pui CH, Yang JJ, Hunger SP, Pieters R, Schrappe M, Biondi A, et al. Childhood acute lymphoblastic leukemia: progress through collaboration. J Clin Oncol. 2015;33:2938-48.

3. Woo JS, Alberti MO, Tirado CA. Childhood B-acute lymphoblastic leukemia: a genetic update. Exp Hematol Oncol. 2014;3:16.

4. He S, Jing CB, Look AT. Zebrafish models of leukemia. Methods Cell Biol. 2017;138:563-92.

5. Jing L, Zon LI. Zebrafish as a model for normal and malignant hematopoiesis. Dis Model Mech. 2011;4:433-8.

6. Langenau DM, Traver D, Ferrando AA, Kutok JL, Aster JC, Kanki JP, et al. Myc- induced $\mathrm{T}$ cell leukemia in transgenic zebrafish. Science. 2003;299:887-90.

7. Feng H, Langenau DM, Madge JA, Quinkertz A, Gutierrez A, Neuberg DS, et al. Heat- shock induction of T-cell lymphoma/ leukaemia in conditional Cre/lox-regulated transgenic zebrafish. Br J Haematol. 2007;138:169-75.

8. Chen J, Jette C, Kanki JP, Aster JC, Look AT, Griffin JD. NOTCH1-induced T-cell leukemia in transgenic zebrafish. Leukemia. 2007;21:462-71.

9. Frazer JK, Meeker ND, Rudner L, Bradley DF, Smith AC, Demarest B, et al. Heritable T-cell malignancy models established in a zebrafish phenotypic screen. Leukemia. 2009;23:1825-35.

10. Gutierrez A, Grebliunaite R, Feng H, Kozakewich E, Zhu S, Guo $\mathrm{F}$, et al. Pten mediates Myc oncogene dependence in a conditional zebrafish model of $\mathrm{T}$ cell acute lymphoblastic leukemia. J Exp Med. 2011;208:1595-603.

11. Feng H, Stachura DL, White RM, Gutierrez A, Zhang L, Sanda T, et al. T-lymphoblastic lymphoma cells express high levels of BCL2, S1P1, and ICAM1, leading to a blockade of tumor cell intravasation. Cancer Cell. 2010;18:353-66.

12. Rudner LA, Brown KH, Dobrinski KP, Bradley DF, Garcia MI, Smith AC, et al. Shared acquired genomic changes in zebrafish and human T-ALL. Oncogene. 2011;30:4289-96.

13. Blackburn JS, Liu S, Wilder JL, Dobrinski KP, Lobbardi R, Moore FE, et al. Clonal evolution enhances leukemia-propagating cell frequency in $\mathrm{T}$ cell acute lymphoblastic leukemia through Akt/mTORC1 pathway activation. Cancer Cell. 2014;25:366-78.

14. Blackburn JS, Liu S, Raiser DM, Martinez SA, Feng H, Meeker $\mathrm{ND}$, et al. Notch signaling expands a pre-malignant pool of T-cell acute lymphoblastic leukemia clones without affecting leukemiapropagating cell frequency. Leukemia. 2012;26:2069-78.

15. Gutierrez A, Feng H, Stevenson K, Neuberg DS, Calzada O, Zhou $\mathrm{Y}$, et al. Loss of function tp53 mutations do not accelerate the onset of myc-induced T-cell acute lymphoblastic leukaemia in the zebrafish. Br J Haematol. 2014;166:84-90.

16. Lobbardi R, Pinder J, Martinez-Pastor B, Theodorou M, Blackburn JS, Abraham BJ, et al. TOX regulates growth, DNA repair, and genomic instability in T-cell acute lymphoblastic leukemia. Cancer Discov. 2017;7:1336-53.

17. Ridges S, Heaton WL, Joshi D, Choi H, Eiring A, Batchelor L, et al. Zebrafish screen identifies novel compound with selective toxicity against leukemia. Blood. 2012;119:5621-31.

18. Gutierrez A, Pan L, Groen RW, Baleydier F, Kentsis A, Marineau $\mathrm{J}$, et al. Phenothiazines induce PP2A-mediated apoptosis in T cell acute lymphoblastic leukemia. J Clin Invest. 2014;124:644-55.
19. Sabaawy HE, Azuma M, Embree LJ, Tsai HJ, Starost MF, Hickstein DD. TEL-AML1 transgenic zebrafish model of precursor B cell acute lymphoblastic leukemia. Proc Natl Acad Sci USA. 2006;103:15166-71.

20. Langenau DM, Ferrando AA, Traver D, Kutok JL, Hezel JP, Kanki JP, et al. In vivo tracking of T cell development, ablation, and engraftment in transgenic zebrafish. Proc Natl Acad Sci USA. 2004;101:7369-74.

21. Anderson NM, Li D, Peng HL, Laroche FJ, Mansour MR, Gjini E, et al. The TCA cycle transferase DLST is important for MYCmediated leukemogenesis. Leukemia. 2016;30:1365-74.

22. Cernan M, Szotkowski T, Pikalova Z. Mixed-phenotype acute leukemia: state-of-the-art of the diagnosis, classification and treatment. Biomed Pap Med Fac Univ Palacky Olomouc Czech Repub. 2017;161:234-41.

23. Traver D, Paw BH, Poss KD, Penberthy WT, Lin S, Zon LI. Transplantation and in vivo imaging of multilineage engraftment in zebrafish bloodless mutants. Nat Immunol. 2003;4:1238-46.

24. Bauer TR Jr., McDermid HE, Budarf ML, Van Keuren ML, Blomberg BB. Physical location of the human immunoglobulin lambda-like genes, 14.1, 16.1, and 16.2. Immunogenetics. 1993;38:387-99.

25. Tang Q, Abdelfattah NS, Blackburn JS, Moore JC, Martinez SA, Moore FE, et al. Optimized cell transplantation using adult rag2 mutant zebrafish. Nat Methods. 2014;11:821-4.

26. Carmona SJ, Teichmann SA, Ferreira L, Macaulay IC, Stubbington MJ, Cvejic A, et al. Single-cell transcriptome analysis of fish immune cells provides insight into the evolution of vertebrate immune cell types. Genome Res. 2017;27:451-61.

27. Tang Q, Iyer S, Lobbardi R, Moore JC, Chen H, Lareau C, et al. Dissecting hematopoietic and renal cell heterogeneity in adult zebrafish at single-cell resolution using RNA sequencing. J Exp Med. 2017;214:2875-87.

28. Novershtern N, Subramanian A, Lawton LN, Mak RH, Haining WN, McConkey ME, et al. Densely interconnected transcriptional circuits control cell states in human hematopoiesis. Cell. 2011;144:296-309.

29. Haferlach T, Kohlmann A, Wieczorek L, Basso G, Kronnie GT, Bene MC, et al. Clinical utility of microarray-based gene expression profiling in the diagnosis and subclassification of leukemia: report from the International Microarray Innovations in Leukemia Study Group. J Clin Oncol. 2010;28:2529-37.

30. Smith AC, Raimondi AR, Salthouse CD, Ignatius MS, Blackburn JS, Mizgirev IV, et al. High-throughput cell transplantation establishes that tumor-initiating cells are abundant in zebrafish Tcell acute lymphoblastic leukemia. Blood. 2010;115:3296-303.

31. Moore FE, Garcia EG, Lobbardi R, Jain E, Tang Q, Moore JC, et al. Single-cell transcriptional analysis of normal, aberrant, and malignant hematopoiesis in zebrafish. J Exp Med. 2016;213:979-92.

32. Palmer C, Diehn M, Alizadeh AA, Brown PO. Cell-type specific gene expression profiles of leukocytes in human peripheral blood. BMC Genom. 2006;7:115.

33. Zhang YA, Salinas I, Li J, Parra D, Bjork S, Xu Z, et al. IgT, a primitive immunoglobulin class specialized in mucosal immunity. Nat Immunol. 2010;11:827-35.

34. Malumbres R, Fresquet V, Roman-Gomez J, Bobadilla M, Robles $\mathrm{EF}$, Altobelli GG, et al. LMO2 expression reflects the different stages of blast maturation and genetic features in B-cell acute lymphoblastic leukemia and predicts clinical outcome. Haematologica. 2011;96:980-6.

35. Kohrer S, Havranek O, Seyfried F, Hurtz C, Coffey GP, Kim E, et al. Pre-BCR signaling in precursor B-cell acute lymphoblastic leukemia regulates PI3K/AKT, FOXO1 and MYC, and can be targeted by SYK inhibition. Leukemia. 2016;30:1246-54. 
36. Lin XC, Liu XG, Zhang YM, Li N, Yang ZG, Fu WY, et al. Integrated analysis of microRNA and transcription factor reveals important regulators and regulatory motifs in adult B-cell acute lymphoblastic leukemia. Int J Oncol. 2017; 50:671-83.
37. Liu X, Li YS, Shinton SA, Rhodes J, Tang L, Feng H, et al. Zebrafish B cell development without a pre-B cell stage, revealed by CD79 fluorescence reporter transgenes. J Immunol. 2017;199:1706-15.

38. Dang CV. MYC on the path to cancer. Cell. 2012;149:22-35. 